\title{
Energy Efficient Target Detection Through Waveform Selection for Multi-Sensor RF Sensing Based Internet of Things
}

\author{
S.Bolisetti ${ }^{\dagger}$, M.Sharma $^{\ddagger}$, M.Patwary ${ }^{\ddagger}$, A.Soliman ${ }^{\dagger}$, E.Benkhelifa ${ }^{*}$ and M.Maguid ${ }^{\S}$ \\ ${ }^{\dagger}$ School of Creative Arts and Engineering, Staffordshire University, Stoke-on-Trent, UK \\ ${ }^{\ddagger}$ School of Computing and Digital Technology, Birmingham City University, Birmingham, UK \\ * School of Computing and Digital Technologies, Staffordshire University, Stoke-on-Trent, UK \\ $\S$ Department of Science and Technology, University Campus Suffolk, Ipswich, UK \\ Email: bolisettisk@ieee.org, \{mak.sharma; mohammad.patwary\}@bcu.ac.uk, a.soliman@staffs.ac.uk, \\ e.benkhelifa@staffs.ac.uk and m.abdel-maguid@uos.ac.uk
}

\begin{abstract}
In this paper, we explore multi-sensor Radio Frequency (RF) sensing based Internet of Things (IoT) for surveillance applications. RF sensing techniques are the next generation technologies which offer distinct advantages over traditional means of sensing. Traditionally, Energy detection (ED) has been used for surveillance applications due to its low computational complexity. However, ED is unreliable due to high false detection rates. There is a need to develop surveillance strategies which offer reliable target detection rates. In this paper, we have proposed a multi-sensor RF sensing based target detection architecture for IoT. To perform surveillance within IoT, multiple sensor nodes are required to co-exist while performing the desired tasks. Interfering waveforms from the neighbouring sensor nodes have a significant impact on the target detection reliability of IoT. In this paper, a waveform selection criterion has been proposed to optimise the target detection reliability and power consumption within IoT in the presence of interfering waveforms.
\end{abstract}

Index Terms-Energy efficiency, internet of things (IoT), multisensor, RF sensing

\section{INTRODUCTION}

Internet of Things (IoT) consist of low-cost, easy-to-deploy network of sensor nodes which are deployed within a predefined sensing region. Once deployed, the sensor nodes coordinate among themselves to perform assigned tasks. The sensor nodes are equipped with one or more sensing devices with limited power, processing, and communication capabilities. Due to low-cost and ease of deployment, IoT have found its place in numerous civilian and military applications $[1,2]$. The sensor nodes can be deployed in hostile and harsh sensing environments and their ability to collectively monitor the sensing region, makes them ideal candidates for surveillance applications [3,4]. Depending on the nature of the sensing application, the sensor nodes can either actively interact with the sensing environment or passively monitor the same. RF sensing based active detection techniques can provide increased reliability and higher target detection rates. The sensor nodes transmit RF signals into the sensing region and detect the presence of targets by observing the received echoes $[5,6]$. Transmission of RF signals by the active sensor nodes is associated with significant increase in the power consumption within the sensor nodes with limited available power. Efficient target detection strategies need to be developed to provide reliable target detection rates with reduced energy consumption.

\section{RELATED WORK}

In the existing literature, various RF sensing based target detectors [7-10] have been proposed by the authors. However, existing RF sensing based target detection strategies are computationally intense for resource constrained sensor nodes within IoT. Recent advancements in UWB $[6,11]$ technologies allowed development of low-cost devices which can transmit relatively short UWB pulses. Due to ultra-short nature of UWB pulses, it is required to transmit significantly lower power for these pulses. This has made RF sensing techniques possible for resource constrained IoT. However, as a consequence of low transmit power and subsequent propagation losses, the received echoes are associated with lower energies. Within the received signal components, detecting the presence of these echoes which are usually corrupted by noise and interference is extremely challenging. The choice of the transmit waveform has a significant impact on the target detection performance of IoT. With multiple sensor nodes deployed within the sensing region, the problem of the waveforms transmitted from the neighbouring sensor nodes interfering with each other is required to be addressed [12]. Conventional multisensor communication techniques are not suitable for RF sensing applications to eliminate such interferences. Within the existing literature, orthogonal waveform design techniques have been considered to allow multiple access [13-15]. However, transmitting these orthogonal waveforms require complex transmitting devices which are usually unavailable within resource constrained wireless sensor nodes. Due to event driven nature of the sensor nodes within IoT, the transmission cycles of independent sensor nodes may not always be synchronised and hence the choice of orthogonal waveforms may not always be the optimal solution. A simple and resource efficient solu- 
tion for the choice of transmit waveforms is required which guarantees reliable target detection rates within the constraints of the given sensing conditions. In this paper, we considered the choice of transmit waveforms which are suitable for RF sensing based applications of IoT. The relevant waveform selection strategies to optimise the target detection reliability within the resource constraints of IoT have been proposed. The main contributions of this paper are,

- RF sensing based target detection architecture for surveillance applications of IoT.

- Suitable transmit waveform selection criterion for application-specific IoT has been proposed

- Target detection optimisation procedure in the presence of interfering waveforms has been proposed.

Rest of the paper is organised as follows: Received signal model and problem formulation for the proposed IoT is discussed in Section III. The transmit waveforms which are suitable of IoT are discussed in Section IV. Hypothesis testing and the proposed target detection optimisation procedure through waveform selection is presented in Section V. In Section VI, we analyse the proposed waveform selection procedure and the performance of the proposed IoT through simulation results which is followed by the conclusion in Section VII

\section{Problem Formulation}

The problem of interest is RF sensing based IoT whose primary objective is to provide surveillance within the sensing region. The proposed IoT consists of clusters of sensor nodes which are distributed within the sensing region. Each cluster consists of a primary node which acts as the cluster-head and a group of receiving nodes. The primary node within each cluster transmits the desired RF signal into the sensing region.

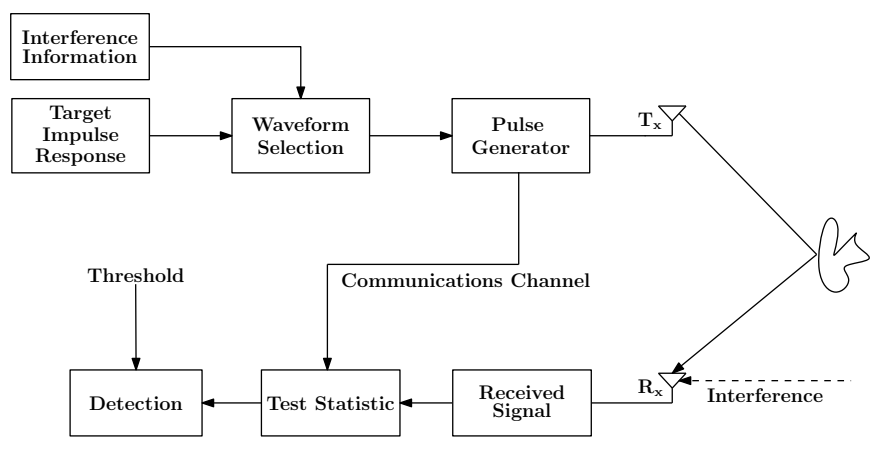

Fig. 1. Proposed Target Detection Model for IoT

As shown in Fig. 1, in the event of existence of a target within the sensing region, the receiving nodes attempt to detect the reflected echoes of the known transmitted signal. The primary node is assumed to have sufficient resources to transmit the desired RF waveforms. To meet the power constraints low-power UWB pulses are transmitted with short transmit durations. As a result of high bandwidth due to ultrashort nature of the transmit pulses, the reflected echoes are characterised by the impulse response of the target. For a target impulse response of length $N_{a}$ and transmit UWB waveform $\mathbf{s}(t)$, the reflected echo from the target can be written as,

$$
\Omega(t)=\mathbf{a}(t) * \mathbf{s}(t)
$$

where $*$ indicates the convolution operator and $\mathbf{a}$ is the target impulse response. It is assumed that IoT has the knowledge of the target's impulse response. In the presence of noise and interfering waveforms, the received target echo at the $i^{\text {th }}$ sensor node can be written as,

$$
\mathbf{y}_{i}(n)=\mathbf{A} \mathbf{s}_{i}(n)+\mathbf{B h}_{i}(n)+\mathbf{w}_{i}(n)
$$

where $\mathbf{y}_{i}$ is the $N_{y} \times 1$ received signal at the $i^{t h}$ sensor node, $\mathbf{s}_{i}$ is the $N_{t} \times 1$ transmitted waveform. A is the $N_{y} \times$ $N_{t}$ convolution matrix for the target impulse response. $\mathbf{h}_{i}$ is interfering waveform and $\mathbf{w}_{i}$ is thermal noise. $\mathbf{B}$ is $N_{y} \times N_{t}$ convolution matrix for the channel impulse response.

$$
\mathbf{A}=\left[\begin{array}{ccccc}
\mathbf{a}(1) & 0 & \ldots & \ldots & 0 \\
\mathbf{a}(2) & \mathbf{a}(1) & \ddots & \ldots & 0 \\
\vdots & \vdots & \ddots & \ddots & 0 \\
\mathbf{a}\left(N_{a}\right) & \mathbf{a}\left(N_{a}-1\right) & \ldots & \mathbf{a}(1) & 0 \\
0 & \mathbf{a}\left(N_{a}\right) & \mathbf{a}\left(N_{a}-1\right) & \ldots & \mathbf{a}(1) \\
\vdots & 0 & \mathbf{a}\left(N_{a}\right) & \ldots & \mathbf{a}(2) \\
\vdots & \vdots & 0 & \ddots & \vdots \\
0 & 0 & \ldots & 0 & \mathbf{a}\left(N_{a}\right)
\end{array}\right]
$$

Here, $N_{y}=N_{t}+N_{a}-1$. The channel convolution matrix $\mathbf{B}$ can be written similarly as shown in (3). The complexity of the convolution matrix is dependent on the impulse response time and independent of the number of interfering sensor nodes. Estimation of channel impulse response has been addressed by the authors in the existing literature $[16,17]$ and in this paper, we assume that an estimate of the channel impulse response is available to the target detector.

\section{TRANSMIT WAVEFORMS}

The choice of an appropriate transmit waveform is an important design parameter for RF sensing based surveillance applications of IoT. To achieve longevity, optimum choice of a transmit waveform within IoT must fulfil the necessary criterion to achieve the desired target detection reliability while operating within the constraints of the available resources. Brevity of the transmit pulses is required to reduce the transmission costs. To reduce the signal processing complexities, the choice of a transmit waveform with good correlation properties is desirable. In this section, we study some of the transmit waveforms which are suitable for IoT and discuss their correlation properties [13-15].

Gaussian pulse is one of the most commonly discussed waveforms. In the time domain, ultra-short Gaussian pulses can be generated which are suitable for resource constrained 
IoT. The time domain expression of a Gaussian pulse can be written as,

$$
\mathbf{g}(t)=\operatorname{Aexp}\left(\frac{-t}{\tau}\right)^{2}
$$

Where $A$ is the maximum amplitude and $\tau$ is the pulse width. The first derivative of the Gaussian pulse gives a Monocycle pulse which is also a commonly discussed waveform. The expression for a Monocycle pulse is,

$$
\mathbf{m}(t)=A\left(\frac{t}{\tau}\right) \exp \left(-\left(\frac{t}{\tau}\right)^{2}\right)
$$

Due to simplicity and ease of generation, the Gaussian and Monocycle pulses can be used within IoT with very low computational cost. However, within a large sensing region with multiple transmitting nodes the target detection rates severely deteriorate due to interference from the neighbouring sensor nodes. Hence a degree of diversity in the choice of waveforms among the transmitting nodes is required.

Orthogonal waveforms generated based on modified Gegenbauer and Hermite polynomials can be used to generate short UWB pulses. The diversity in the available waveforms provides a simple solution to allow multiple access. Gegenbauer polynomials are defined in the interval $[-1,1]$. The recurrence equation for the $n^{\text {th }}$ order Gegenbauer polynomial is written as,

$$
\begin{aligned}
\mathcal{G}_{n}(\beta, t)= & \frac{2(n+\beta-1)}{n} t G_{n-1}(\beta, t)- \\
\mathcal{G}_{0}(\beta, t) & =1
\end{aligned}
$$

where $n$ is the order of the Gegenbauer polynomial and $\beta$ is the shape parameter. Modified Gegenbauer polynomials use a weight function $\mathrm{w}(\mathrm{t})$ to facilitate the generation of ultra-short pulses. Modified Gegenbauer functions are written as,

$$
\begin{aligned}
\mathbf{G}(\beta, t) & =\sqrt{\mathbf{w}(t)} \mathcal{G}_{n}(\beta, t) \\
\mathbf{w}(t, \beta) & =\left(1-t^{2}\right)^{\beta-\frac{1}{2}} \quad \beta>\frac{-1}{2}
\end{aligned}
$$

Modified Hermite polynomials are defined in the interval $[-\infty, \infty] . n^{t h}$ order Hermite polynomial can be written as,

$$
\begin{aligned}
& h_{e_{n}}(t)=(-1)^{n} e^{\frac{t^{2}}{2}} \frac{d^{n}}{d t^{n}}\left(e^{\frac{-t^{2}}{2}}\right) \quad n \neq 0 \\
& h_{e_{0}}(t)=1
\end{aligned}
$$

Modified Hermite polynomials are obtained by multiplying Hermite polynomials with $e^{\frac{-t^{2}}{4}}$. A $n^{t h}$ order Modified Hermite function is,

$$
h_{n}(t)=e^{\frac{-t^{2}}{4}} h_{e_{n}}(t)
$$

The transmit waveforms discussed in this section are easy to generate and does not require complex transmitting devices. They also provide diversity within the choice of transmit waveforms. These proposed waveforms which are suitable for UWB communications, can be transmitted as short pulses with reduced transmission costs. However, due to event driven nature of IoT and spatial displacement of the sensor nodes, orthogonal waveforms do not provide a generalised solution for multiple access.

\section{TARget Detection Optimisation}

The performance measure of an IoT as a surveillance system while dedicated to detecting the existence or non-existence of targets is the degree of reliability on such decision-making process. The two possible outcomes of this decision-making process are hypothesis $H_{0}$ and hypothesis $H_{1}$ which are modelled as a binary hypothesis testing problem. Hypothesis $H_{0}$ represents absence of a target and hypothesis $H_{1}$ represents the existence of a target. The possible received signal models corresponding to hypothesis $H_{0}$ and $H_{1}$ are,

$$
\begin{aligned}
& H_{0}: \begin{cases}H_{00}: & \mathbf{y}(n)=\mathbf{w}(n) \\
H_{01}: & \mathbf{y}(n)=\mathbf{B h}(n)+\mathbf{w}(n)\end{cases} \\
& H_{1}: \begin{cases}H_{10}: & \mathbf{y}(n)=\mathbf{A} \mathbf{s}(n)+\mathbf{w}(n) \\
H_{11}: & \mathbf{y}(n)=\mathbf{A} \mathbf{s}(n)+\mathbf{B h}(n)+\mathbf{w}(n)\end{cases}
\end{aligned}
$$

(11) and (12) represent possible received signal models under hypothesis $H_{0}$ and $H_{1}$ which are characterised by existence and absence of the target and the interfering waveforms. A Cross-Correlation (CC) based detector is a popular technique which is used to detect the existence of known waveforms within the received signal component. A CC detector performs cross-correlation between the received signal and the target signal to make a decision regarding the existence of the desired waveform.

$$
r(\Delta T)=\sum_{n=-\infty}^{\infty} \mathbf{s}(n-\Delta T) \mathbf{y}(n)
$$

Where, $\Delta T$ is the time delay corresponding to a given range bin. However, as a consequence of the ultra-wide band nature of the transmit signal, the reflected echoes undergo shape transformation. Hence the optimal detector should match the scattered waveform in (1) with the received signal. If matched filter impulse response is $\mathbf{f}$, then the matched filtered output of the received signal is given by,

$$
\mathbf{x}=\mathbf{f}^{H} \mathbf{y}=\mathbf{f}^{H} \mathbf{A} \mathbf{s}+\mathbf{f}^{H} \mathbf{B} \mathbf{h}+\mathbf{f}^{H} \mathbf{w}
$$

Signal to Interference and Noise Ratio (SINR) at the matched filter output is given by,

$$
S I N R=\frac{\left|\mathbf{f}^{H} \mathbf{A} \mathbf{s}\right|^{2}}{E\left[\left|\mathbf{f}^{H} \mathbf{B h}\right|^{2}\right]+E\left[\left|\mathbf{f}^{H} \mathbf{w}\right|^{2}\right]}
$$

From [18], the matched filter impulse response that maximises the SINR at the receiver can be written as,

$$
\mathbf{f}=\alpha\left(\mathbf{B h h}^{H} \mathbf{B}^{H}+\mathbf{R}_{w}\right)^{-1} \mathbf{A} \mathbf{s}
$$


Where, $\alpha$ is a scaling parameter and $\mathbf{R}_{w}$ is the noise covariance matrix. However, the matched filter in (16) only produces the maximum attainable SINR under the constraints of the transmitted waveform. From (14) and (16), the optimum transmit waveform that maximises the target return at the matched filter output can be written as,

$$
\hat{\mathbf{s}}=\max _{\mathbf{s}} \mathbf{s}^{H} \mathbf{A}^{H}\left(\mathbf{B h h}^{H} \mathbf{B}^{H}+\mathbf{R}_{w}\right)^{-1} \mathbf{A} \mathbf{s}
$$

The solution to (17) can be obtained through eigen-analysis. The transmit waveform that maximises the matched filter output is the eigenvector corresponding to the maximum eigenvalue of $\mathbf{A}^{H}\left(\mathbf{B h h}^{H} \mathbf{B}^{H}+\mathbf{R}_{w}\right)^{-1} \mathbf{A}$. However, the optimised waveform obtained in (17) is associated with transmission complexities and the resource constrained sensor nodes within the IoT may not have sufficient resources to transmit these waveforms. To reduce the transmission complexity, we consider the transmit waveforms discussed in Section IV. While the waveforms discussed in Section IV do not optimise the matched filter output, suitable waveform may be chosen which maximises the matched filter output within the constraints of the available transmit waveforms. However, maximising SINR is not the sufficient condition to optimise the target detection performance of IoT.

The matched filter compares the received signal with the expected target return to detect the existence of the target echo. A reliable decision regarding the existence or absence of a target can be made when the matched filter outputs under hypothesis $H_{0}$ and $H_{1}$ are clearly distinguishable. To measure the ability of the matched filter to make this distinction we define Ease of Detection Index $(\delta)$ in $(18)$ which is measured at $3 \mathrm{~dB}$ Signal-to-Interference Ratio (SIR). Here $\star$ represents correlation operator. When the sensing conditions are known, the transmit waveform which maximises $(\delta)$ gives optimum reliability among the available choice of transmit waveforms. Within resource constrained IoT, the transmit power has a significant impact on the life time of the sensor nodes. The choice of transmit waveform while achieving high $\delta$, must also be energy efficient to ensure longevity of the sensor nodes. The amount of transmit power required to guarantee a desired SIR at the receiver is related to various factors such as propagation losses, target impulse response, target range, etc. For given sensing conditions, the energy efficiency of the transmit waveform is defined by Energy Efficiency Index $(\eta)$ which is the ratio of the amount of transmit power $\left(P_{T m}\right)$ required to guarantee a desired SIR at the matched filter output to the amount of transmit power $\left(P_{T r}\right)$ required to guarantee the desired SIR at the sensor node receiver which is given as,

$$
\begin{aligned}
\eta=\frac{P_{T m}}{P_{T r}} & =\frac{\max \left(\mathbf{f}^{H} \star \mathbf{B h}\right)(\mathbf{A} \mathbf{s})^{H}(\mathbf{A} \mathbf{s})}{\max \left(\mathbf{f}^{H} \star \mathbf{A} \mathbf{s}\right)(\mathbf{B h})^{H}(\mathbf{B h})} \quad \text { (19) } \begin{array}{l}
\text { received signal models discussed in }(11) \\
\text { cates the received signal model under hyp }
\end{array} \\
\delta & =\frac{\left(\max \left(\mathbf{f}^{H} \star \mathbf{A} \mathbf{s}\right)-\max \left(\mathbf{f}^{H} \star \mathbf{B h}\right)\right)\left(\max \left(\mathbf{f}^{H} \star(\mathbf{A} \mathbf{s}+\mathbf{B h})\right)-\max \left(\mathbf{f}^{H} \star \mathbf{B h}\right)\right)}{\max \left(\mathbf{f}^{H} \star \mathbf{B h}\right)}
\end{aligned}
$$

Energy efficiency index denotes the factor by which the transmit power may be reduced while ensuring desired SIR at the matched filter output. The transmit waveform which provides optimal balance between $\delta$ and $\eta$ is chosen based on the criterion given by the ratio $\delta / \eta$. Therefore, for given sensing conditions the transmit waveform which maximises $\delta / \eta$ optimises the target detection reliability of IoT.

\section{Performance AnAlysis}

In this section, we compare the optimality criterion of the waveforms discussed in Section IV. Simulations are performed in MATLAB and within simulations, the interfering waveform is assumed to be a Monocycle pulse and the presence of white Gaussian noise is assumed. IoT is assumed to be deployed to detect the presence of a target with known impulse response. In Table I, $\delta$ and $\eta$ of the waveforms considered under the given sensing conditions are summarised. High value of Ease of Detection Index indicates greater target detection reliability and lower value for Energy Efficiency Index indicates improved transmission efficiency. From Table I it can be observed that for the simulated sensing conditions, $\mathrm{G}_{4}$ and $\mathrm{Hm}_{4}$ waveforms generated high $\delta / \eta$ ratios compared to the other waveforms. Similarly, $\delta / \eta$ ratio of $H m_{1}$ waveform is less than zero which indicates that for the given sensing conditions $\mathrm{Hm}_{1}$ waveform is unsuitable for transmission.

TABLE I

Comparison of GAUSSiAn AND Monocycle Pulses AUTOCORRELATION FUNCTIONS

\begin{tabular}{cccc}
\hline Waveform & $\delta$ & $\eta$ & $(\delta / \eta)$ \\
\hline Gaussian Pulse & 0.4553 & 0.2277 & 1.9995 \\
\hline$G_{0}$ & 0.4045 & 0.2214 & 1.8272 \\
\hline$G_{1}$ & 0.3906 & 0.3789 & 1.0309 \\
\hline$G_{2}$ & 0.0357 & 0.5609 & 0.0636 \\
\hline$G_{3}$ & 0.4180 & 0.1440 & 2.9024 \\
\hline$G_{4}$ & 1.2945 & 0.0843 & 15.3645 \\
\hline$H m_{0}$ & 0.1969 & 0.4029 & 0.4888 \\
\hline$H m_{1}$ & -0.0333 & 1.3813 & -0.0241 \\
\hline$H m_{2}$ & 0.3603 & 0.2523 & 1.4281 \\
\hline$H m_{3}$ & 1.2444 & 0.0958 & 12.9957 \\
\hline$H m_{4}$ & 1.2675 & 0.0857 & 14.7904 \\
\hline
\end{tabular}

Matched Filter Output: In Fig. 2 and Fig. 3, the matched filter outputs of the received signal models under hypothesis $H_{0}$ and $H_{1}$ with transmit waveforms being $\mathrm{G}_{4}$ and $\mathrm{Hm}_{4}$ respectively are plotted. The transmission periods of individual sensor nodes within IoT are assumed to be unsynchronised. The matched filter outputs are plotted for all 4 cases of received signal models discussed in (11) and (12). $H_{00}$ indicates the received signal model under hypothesis $H_{0}$ where 
the target and interfering waveforms are absent. Similarly, $H_{01}$ refers to the received signal under hypothesis $H_{0}$ in the presence of interfering waveform. Similarly, $H_{10}$ and $H_{11}$ refer to the received signal models under hypothesis $H_{1}$ in the absence and presence of interfering waveform. From the plots, it can be observed that, when $\mathrm{G}_{4}$ and $\mathrm{Hm}_{4}$ waveforms are transmitted respectively, a clear distinction existed between matched filter outputs under hypothesis $H_{0}$ and $H_{1}$ which indicates greater detection reliability.

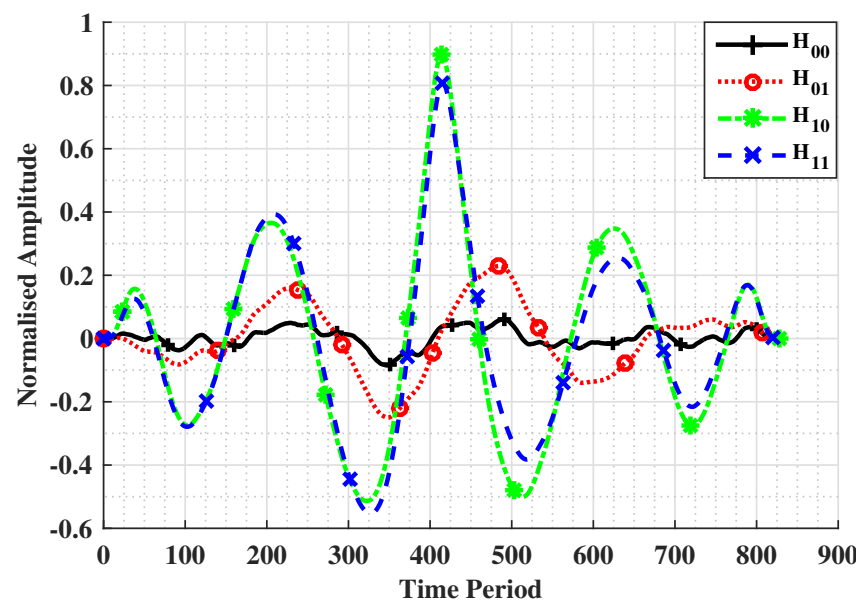

Fig. 2. Matched filter output for $G_{4}$ transmit waveform and interfering Monocycle pulse

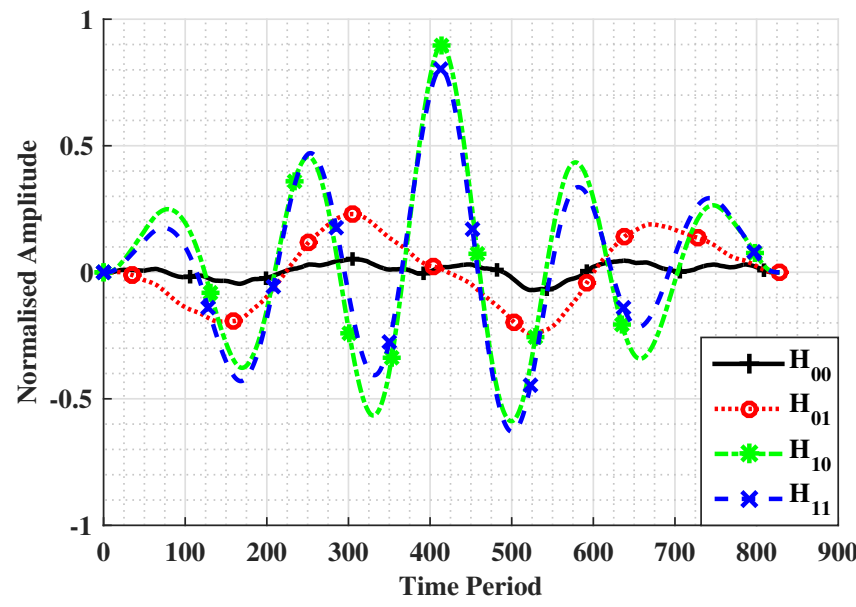

Fig. 3. Matched filter output for $\mathrm{Hm}_{4}$ transmit waveform and interfering Monocycle pulse

Similarly, in Fig. 4, the matched filter output is plotted when $G_{3}$ waveform is transmitted. In this case at $3 \mathrm{~dB}$ SINR, while the matched filter output is maximised under hypothesis $H_{1}$, a reduced distinction can be observed between hypothesis $H_{0}$ and $H_{1}$ along with lower dynamics. This leads to reduced detection reliability. In Fig. 5, the matched filter output is plotted when $\mathrm{Hm}_{1}$ waveform is transmitted. In this case, no distinction between matched filter outputs under hypothesis
$H_{0}$ and $H_{1}$ can be observed which indicates an uncertainty in the decision-making process.

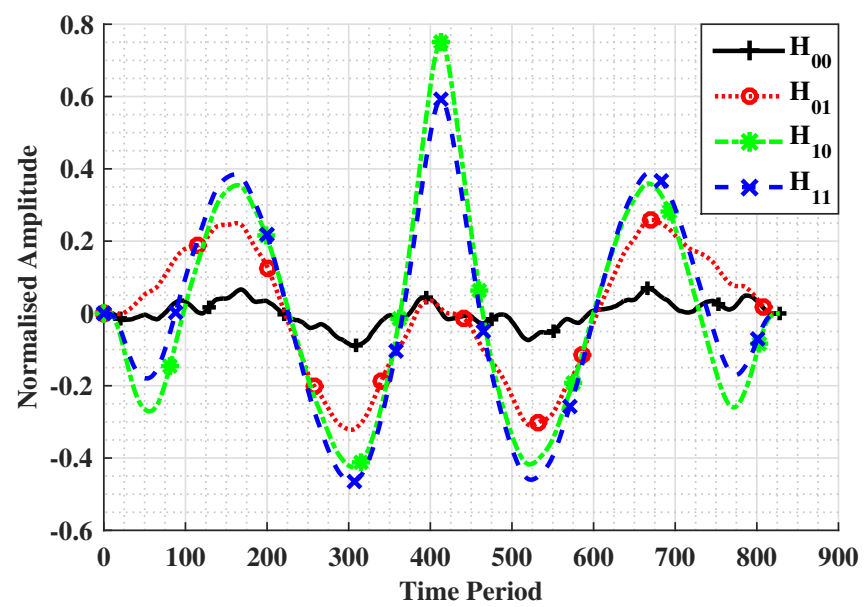

Fig. 4. Matched filter output for $G_{3}$ transmit waveform and interfering Monocycle pulse

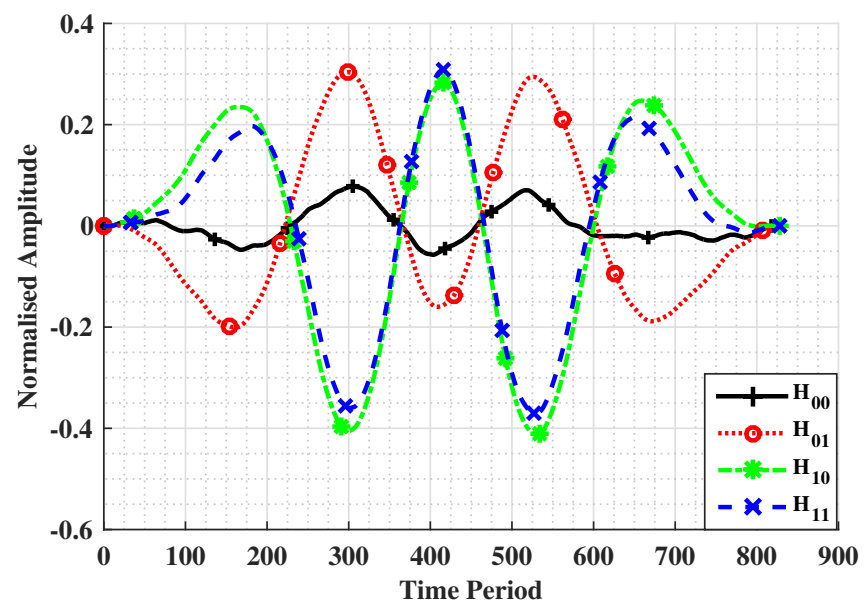

Fig. 5. Matched filter output for $H m_{1}$ transmit waveform and interfering Monocycle pulse

Target Detection Performance: To make a decision regarding the existence of targets, a test statistic $\mathbb{T}$ is generated based on likelihood ratio test which is compared to a pre-defined threshold $\gamma$. The test statistic for the detection problem based on matched filter output can be written as [19]:

$$
\mathbb{T}=\frac{\mathbf{f}^{H} \mathbf{y}}{\mathbf{y}^{H} \mathbf{y}-\mathbf{f}^{H} \mathbf{y}} \stackrel{H_{H_{0}}}{\gtrless} \gamma
$$

$\gamma$ is usually chosen such that the maximum false alarm rate $\left(P_{f a}\right)$ remains within an acceptable limit. For simulations, $\gamma$ is chosen to ensure a maximum allowable $P_{f a}$ of $10^{-3}$. We resort to Monte-Carlo techniques for simulations with $10 / P_{f a}$ independent simulations with $N_{y}=415$ received signal samples. The target impulse response vector length 
$N_{a}$ is assumed to be 15. A traditional Energy detector (ED) is simulated to compare the detection reliability and power efficiency of the proposed method [20]. According to the results summarised in Table I and simulation results for the matched filter outputs discussed in this section, within the given sensing conditions, $G_{4}$ waveform is expected to provide optimum target detection performance. To verify the observations, in Fig. 6 we compare the target detection performances of the proposed target detector when $G_{4}$ and $H m_{1}$ waveforms are transmitted respectively. Clearly, when $G_{4}$ waveform is transmitted the target detector outperformed its counterpart when $\mathrm{Hm}_{1}$ waveform is used. Moreover, traditional ED also outperformed target detection performance of the detector when $\mathrm{Hm}_{1}$ waveform is used.

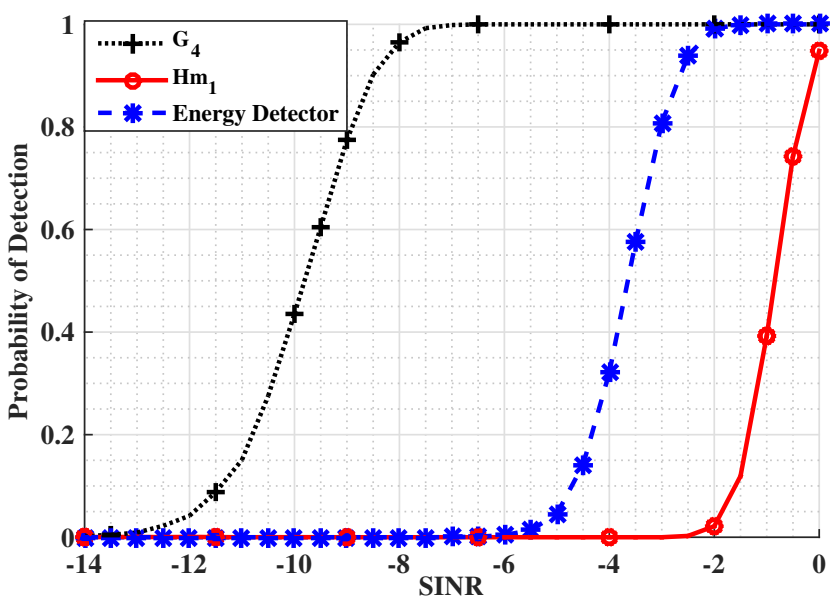

Fig. 6. Comparison of target detection performance when $G_{4}$ and $H m_{1}$ waveforms are transmitted

Similarly, in Fig. 7, the target detection performances are compared when $G_{4}$ and $G_{3}$ waveforms are transmitted. While $G_{3}$ provided a significant improvement in target detection performance when compared to $H m_{1}, G_{4}$ outperformed $G_{3}$ by a significant margin. However, target detection using $G_{3}$ waveform outperformed the corresponding performance of traditional ED. Finally, in Fig. 8, the target detection performances are compared when $G_{4}$ and $\mathrm{Hm}_{4}$ waveforms are transmitted. From Table I it can be observed that the difference between $\delta / \eta$ ratios of $G_{4}$ and $\mathrm{Hm}_{4}$ waveforms are comparable which is validated through simulations in Fig. 8 with $G_{4}$ slightly outperforming $\mathrm{Hm}_{4}$.

Within Eq. 20, the amount of transmit power, $P_{T m}$ required to guarantee a desired SIR at the matched filter output is measured as,

$$
P_{T m}=S I R^{2}\left(\frac{\max \left(\mathbf{f}^{H} \star \mathbf{B h}\right)}{\max \left(\mathbf{f}^{H} \star \mathbf{A s}\right)}\right)^{2}
$$

At $0.5 \mathrm{~mW}$ interfering signal strength, the power consumption of the transmit UWB waveforms based on the proposed waveform selection criterion is plotted in Fig. 9. Power re-

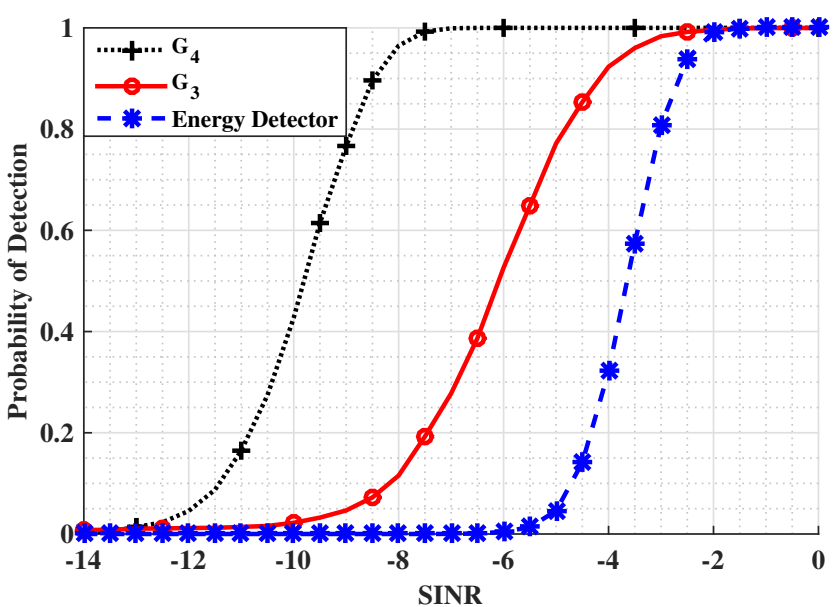

Fig. 7. Comparison of target detection performance when $G_{4}$ and $G_{3}$ waveforms are transmitted

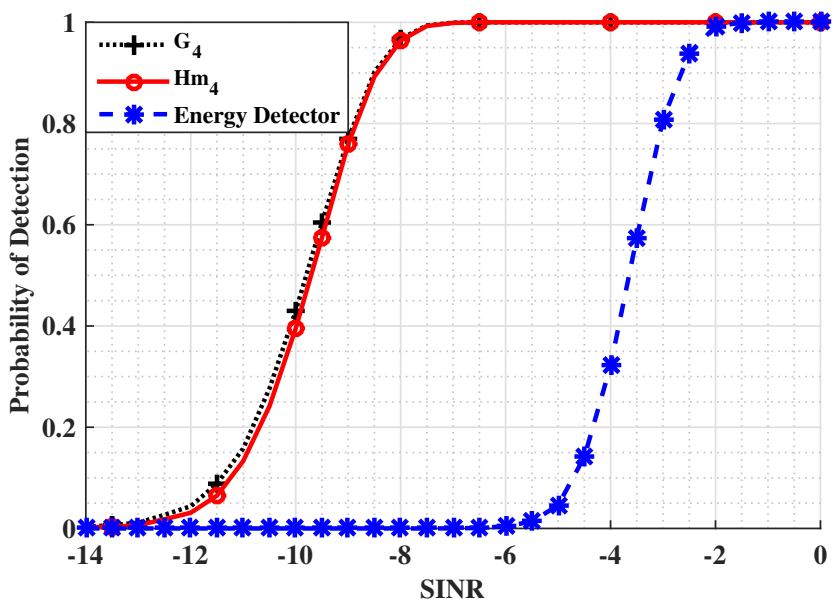

Fig. 8. Comparison of target detection performance when $G_{4}$ and $H m_{4}$ waveforms are transmitted

quirements of an energy Detector is provided for reference. It can be observed that, the transmit waveforms selected based on the proposed selection criterion significantly reduces the power consumption within resource constrained IoT. Among the selected waveforms, $\mathrm{G}_{4}$ and $\mathrm{Hm}_{4}$ consumed least amount of power followed by $G_{3}$ and $H m_{1}$. The results plotted in Fig. 9 validate the proposed Energy Efficiency Index $(\eta)$ which is summarised in Table. I. Hence, with known target impulse response and sensing conditions, the waveform selection procedure is performed considering amount of power consumption as well as maximum allowable $P_{f a}$. The proposed framework to measure the $\delta / \eta$ ratio is expected to provide with an indicative benchmark on the suitability of a certain waveform. To calibrate a detection system the highest possible value of such threshold is to be preferred. 


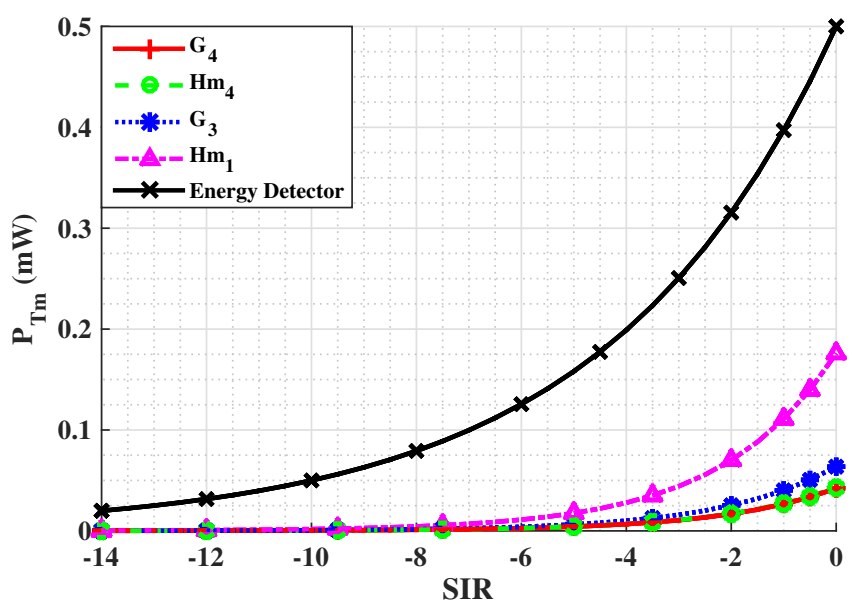

Fig. 9. Energy efficiency of the proposed waveform selection criterion

\section{CONCLUSION}

We have investigated the waveform selection criterion in the context of energy efficiency and detection reliability within RF sensing based IoT for surveillance applications. The amount of power consumed during RF signal transmission is one of the major disadvantages of RF sensing when considered for low-powered device based IoT. Transmit waveforms within the range of UWB technologies which are suitable for resource constrained IoT have been explored. Various received signal models in the context of multi-sensor UWB based IoT have been discussed. Within an event driven IoT, where the transmission periods of individual sensor nodes are unsynchronised, we proposed a waveform selection criterion to optimise the target detection reliability. The proposed waveform selection criterion takes into account the nature of the target and sensing conditions to generate ease of detection index and energy efficiency index for all available transmit waveforms. Numerical results show the target detection performance and energy efficiencies of the proposed IoT for different choices of transmit waveforms. It has been shown that the proposed waveform selection criterion optimises the target detection reliability as well as the energy efficiency under the constraints of available choices of transmit waveforms. In the future work, authors consider deriving a reconfigurable and dynamic waveform selection feature for the existing GLRT detector to achieve increased the detection reliability while meeting the resource constraints of IoT.

\section{REFERENCES}

[1] D. Estrin, R. Govindan, J. Heidemann, and S. Kumar, "Next century challenges: Scalable coordination in sensor networks," in Proceedings of the 5th annual ACM/IEEE international conference on Mobile computing and networking, 1999, pp. 263-270.

[2] A. Arora, P. Dutta, S. Bapat, V. Kulathumani, H. Zhang, V. Naik, V. Mittal, H. Cao, M. Demirbas, M. Gouda, Y. Choi, T. Herman, S. Kulkarni, U. Arumugam, M. Nesterenko, A. Vora, and M. Miyashita, "A line in the sand: a wireless sensor network for target detection, classification, and tracking," Computer Networks, vol. 46, no. 5, pp. 605 - 634, 2004, military Communications Systems and Technologies.
[3] M. Ali, A. Bhm, and M. Jonsson, "Wireless sensor networks for surveillance applications - a comparative survey of mac protocols," in The Fourth International Conference on Wireless and Mobile Communications, July 2008, pp. 399-403.

[4] F. T. Jaigirdar and M. M. Islam, "A new cost-effective approach for battlefield surveillance in wireless sensor networks," in International Conference on Networking Systems and Security (NSysS), Jan 2016, pp. $1-6$.

[5] P. K. Dutta, A. K. Arora, and S. B. Bibyk, "Towards radar-enabled sensor networks," in 5th International Conference on Information Processing in Sensor Networks, April 2006, pp. 467-474.

[6] M. Ditzel and F. H. Elferink, "Low-power radar for wireless sensor networks," in European Radar Conference, Sept 2006, pp. 139-141.

[7] S. K. Bolisetti, K. Ahmed, M. Patwary, and M. Abdel-Maguid, "Compressive parametric glrt detector for airborne mimo radar," in International Conference on Wireless Communications and Signal Processing (WCSP), Nov 2011, pp. 1-5.

[8] N. Pulsone and M. Zatman, "A computationally efficient two-step implementation of the GLRT," IEEE Transactions on Signal Processing, vol. 48, no. 3, pp. $609-616$, Mar 2000.

[9] E. Conte, A. De Maio, and G. Ricci, "GLRT-based adaptive detection algorithms for range-spread targets," IEEE Transactions on Signal Processing, vol. 49, no. 7, pp. 1336 -1348, Jul 2001.

[10] S. K. Bolisetti, M. Patwary, K. Ahmed, A.-H. Soliman, and M. AbdelMaguid, "Subspace compressive glrt detector for mimo radar in the presence of clutter," The Scientific World Journal, vol. 2015, 2015.

[11] "The samraksh company. user manual for the bumblebee: A low-power, mote-scale pulsed doppler radar sensor board," https://samraksh.com/index.php/products/sensors/32-productpages/products-sensors/71-bumblebee-radar, (Accessed on: 18-102016).

[12] L. Sakkila, A. Rivenq, C. Tatkeu, Y. ElHillali, F. Boukour, and J. M. Rouvaen, "Comparison of classical and orthogonal uwb waveforms for radar applications," in 6th International Colloquium on Signal Processing its Applications, May 2010, pp. 1-5.

[13] F. Elbahhar, A. Rivenq-Menhaj, J. M. Rouvaen, M. Heddebaut, and T. Boukour, "Comparison between ds-cdma and modified gegenbauer functions for a multiuser communication ultra-wideband system," IEE Proceedings - Communications, vol. 152, no. 6, pp. 1021-1027, Dec 2005.

[14] L. B. Michael, M. Ghavami, and R. Kohno, "Multiple pulse generator for ultra-wideband communication using hermite polynomial based orthogonal pulses," in IEEE Conference on Ultra Wideband Systems and Technologies, May 2002, pp. 47-51.

[15] L. Sakkila, A. Rivenq, C. Tatkeu, Y. Elhillali, J.-P. Ghys et al., "Performances of micropower uwb radar using orthogonal waveforms," Wireless Engineering and Technology, vol. 5, no. 03, p. 74, 2014.

[16] T. Aittomki and V. Koivunen, "Improved mimo radar channel estimation using spatial coding," in IEEE International Conference on Acoustics, Speech and Signal Processing, May 2013, pp. 4120-4124.

[17] H. Hashemi, "Impulse response modeling of indoor radio propagation channels," IEEE Journal on Selected Areas in Communications, vol. 11, no. 7, pp. 967-978, Sep 1993.

[18] C. Y. Chen and P. P. Vaidyanathan, "Mimo radar waveform optimization with prior information of the extended target and clutter," IEEE Transactions on Signal Processing, vol. 57, no. 9, pp. 3533-3544, Sept 2009.

[19] J. Liu, W. Liu, B. Chen, H. Liu, and H. Li, "Detection probability of a cfar matched filter with signal steering vector errors," IEEE Signal Processing Letters, vol. 22, no. 12, pp. 2474-2478, Dec 2015.

[20] N. Armi, B. A. W. Chaeriah, D. Mahmudin, P. Daud, and M. Arshad, "Energy detector threshold under white gaussian noise channel in cognitive radio system," in 2015 2nd International Conference on Information Technology, Computer, and Electrical Engineering (ICITACEE), Oct 2015, pp. 372-375. 